\title{
SIX SIGMA CONTROL LIMITS AND A FEW APPLICATIONS
}

\author{
Ambika C., ${ }^{*}$ Prakash R. ${ }^{* *} \&$ Nagaraja Rao C. ${ }^{* * *}$
}

\begin{abstract}
Sigma $(\sigma)$ is a Greek letter that has become the statistical symbol and mefric of process variation. Six sigma is a disciplined, customers focused process designed to help organizations to move towards the creation of near perfect products and services. Six sigma $(6 \sigma)$ increases quality by reducing variation, defects and costs. $6 \sigma$ is used in almost all fields of humari activity. $6 \sigma$ has been considered a powerful business strategy that employs a well structured continuous improvement methodology to reduce process variability and drive out waste within the business processes using effective opplication of statistical tools and techniques. This article i) gives the general introduction about the concept of six sigma, ii) briefs about the two $6 \sigma$ methodologies DMAIC and DMADV, which are most commonly used by the orgonizations to achieve $6 \sigma$ performance level, iii) discusses some of the applications of these limits in reducing the process variability. Some remarks about the results of applications of $6 \sigma$, the future requirements and benefits of $6 \sigma$ are also discussed.
\end{abstract}

* Department of Statistics, Mount Carmel College, Bangalore.

** Department of Statistics, Vijaya College, Bangalore.

***Department of Statistics, Vijaya College, Bangalore. 


\section{Introduction}

Sigma $(\sigma)$ is a Greek alphabet and the sigma value indicates how offen defects are likely to occur. Six sigma's target is to achieve less than 3.4 defects or errors per million opportunities. Higher the number of sigmas, the more consistent the process output or smaller the variation is. If the specification limits are $6 \sigma$ above and below the process mean, then defects will not be more than 3.4 per million opportunities and such a product characteristic and the process that produced it is said to be six sigma. Six sigma is a disciplined, customers focused process designed to help organizations to move towards the creation of near perfect products and services. Six is the number of sigmas measured in a process when the variation around the target is such that only 3.4 outputs out of one million are defects under the assumption that the process average may drift over the long term by as much as 1.5 standard deviations. A defect in the context of $6 \sigma$ is defined as anything that does not meet the customer requirements. 60 implementation in any organization would eliminate almost all defects, rework and scrap. This would lead to processes under statistical control and quality is based on design rather than inspecting for defects at the end of the process. $6 \sigma$ is used in almost all fields, like service sector, healthcare, business management, finance, manufacturing, marketing and other organizations. $6 \sigma$ is a method of preventing defects. It is a statistical measure and a smarter way to manage a business. Six sigma has been considered a powerful business strategy that employs a well structured continuous improvement methodology to reduce process variability and drive out waste within business processes using effective application of statistical tools and techniques.

Also, six sigma is one of the strategies and tools which leading organizations have started using to achieve accuracy and speed and at the same time reduce cost and increase customer satisfaction and profits. 60 has become a strong quality philosophy which links customer requirements and process improvements with financial results while simultaneously providing the desired speed, accuracy and agility in today's e-age. The main objective of this article is to statistically review some of the applications of $6 \sigma$ in reducing process variability. The parts per million defectives with the assumption that the process average may drift over the long term by as much as 1.5 standard deviations is given in the following table. 


\begin{tabular}{|c|c|c|}
\hline Specification limit & Inside specification & ppm defective \\
\hline $\pm 1 \sigma$ & $30.23 \%$ & 697700 \\
\hline $\pm 2 \sigma$ & $69.13 \%$ & 308700 \\
\hline $\pm 3 \sigma$ & $93.32 \%$ & 66800 \\
\hline $\pm 4 \sigma$ & $99.3790 \%$ & 6210 \\
\hline $\pm 5 \sigma$ & $99.9767 \%$ & 233 \\
\hline $\pm 6 \sigma$ & $99.99966 \%$ & 3.4 \\
\hline
\end{tabular}

From the table we can see that as the sigma level is increased the defects per million opportunities decreases exponentially.

\section{Six Sigma Methodology}

A stated sigma level, such as $6 \sigma$ is used to describe how well the process variation meets the customers requirements. Once it is defined, the $6 \sigma$ techniques can be applied to methodically reduce the error rate to improve customer satisfaction. The following is the description of two standard methodologies of $6 \sigma$ control limits.

\section{a) DMAIC methodology}

A standard methodology, DMAIC is an acronym for define, measure, analyze, improve, control. This methodology is designed to Define the problem, Measure the extent of the problem, Analyze the sources of variation, Improve the process and Control the process for sustained improvement. This methodology should be applied from leadership levels of the organization down to the process level. The DMAIC process is an improvement system for existing processes falling below specification and looking for incremental improvement. There are several stages in this. They are:

Define stage: The starting point for the DMAIC problem solving methodology is the define stage. The key objectives are project definition, top level process definition, team formation. The key project details are documented in the project charter which provide a means to control and manage $6 \sigma$ projects. The top level process definition provides a broad view of the process. Team formation is a crucial step for building stockholders buy in. The team members have responsibility to ensure team performance. 
Measure stage: The key objectives in this are process definition, metric definition, process baseline estimation, measurement system analysis. Process definition is to understand the decision points and detailed functionality within the process. Metric definition is to verify a reliable means of process estimation. A process metric is simply a measured parameter from a process that provides some indication of the process state or condition. Process baseline estimation is to clarify the starting point of the project, which provides an estimate of the current state of the process and its ability to meet customer requirements. Measurement systems analysis is to quantify the errors associated with the metric.

Analyze stage: Here objectives are, analysis of value stream, analysis of sources of variation, determining process drivers. The term value stream refers to the necessary activities that contribute value to the product or service as determined by the customer. If sources of variation are found in process, then some focusing tools are used to reduce it. The process drivers are identified that contribute to process variation.

Improve stage: Defining the new process, assessing benefits of proposed solutions, evaluating process failure modes, implementation and verfication are a few important objectives of this stage. The most obvious outcome is the definition of new practices to replace the current operating procedures. Assessing benefits of proposed solutions to assure the stakeholders receive their optimal return on the project investment. Once the process flow is established it can be evaluated for its failure modes, which allows us to define mitigation strategies to minimize the impact or occurrence of failures. Once the improvement methodology and new process levels have been determined, they can be implemented.

Control stage: The objectives are standardize on the new methods, measure bottom line impact, document lessons learned. The methods for process control include statistical process control, engineering process control, and operational procedures. Control plan provides an overview of the strategies that will be used to ensure that key process or part characteristics are controlled through either detection or prevention strategies or the combination of the two. A key part of the control stage is to continuously monitor the effects of the process improvement. The results of the six sigma project should be documented in a project report.

\section{b) DMADV methodology}

The DMADV methodology used in product designiareas and is the acronym used in conjunction with the DFSS (Design for Six Sigma) nomenclature. DMADV is designed with define, measure, analyze, design, verify steps. A design for six sigma DMADV methodology is used to create a new process. The following are the different stages: 
Define stage: Identify the new product, service or process to be designed and determine the project CTQs, problems and goals.

Measure stage: Define the ongoing measurement system. Translate customer needs and requirements into measurable characteristics (CTQs)

Analyze stage: The key steps are developing alternative solutions, building on its knowledge, considering ideas, analyzing trade-offs and selected designs, performing a benchmark analysis.

Design stage: To have a detailed system that met the customer CTQs and which was statistically validated and optimized on the available data.

Verify stage: It requires that designers test and validate the performance of the system developed in the design phase, build a control plan to ensure ongoing optimal performance of the system and document and transition the system so that the customer owns the new design and is able to fully support it.

\section{Tools and Applications}

Six sigma has been effectively used to reduce waste in a coating process and to achieve substantial financial benefits (Ricardo Banuelas et al., 2005). The primary objective here is waste reduction. The project selected is identifying, quantifying and eliminating the source of variation that leads to failure due to spindle changes by the re-winder machine. In the define phase, the defect is defined as the failure of the re-winder to change from one spindle to the other. The potential impact of the project is estimated. The financial benefits are identified and calculated. Measure phase has the purpose of mapping the current process and establishing metrics that describe the project in order to narrow the problem to its major factors. The metric established for the re-winder's performance is a passed or failed chop over. Different tools were used to illustrate the various causes that affect the re-winder performance. The purpose of analyze phase is to start learning about data in order to generate, segment, prioritize and verify the possible root causes and their relationship to the project or outputs. From the information obtained the team decided to concentrate on the gap between the core and the knife. In the improve phase the specification limits of the CTQ characteristic gap was identified. The best alternative is implemented for improving the process, achieved validation of the improvement. In the control phase the proposed solution consists of installing spirit levels in the extreme of the turret to measure the angle and therefore the gap between the turret and the arm. The material waste was reduced nearly by 50000 per year. As a result a significant financial benefit was achieved in a relatively short period of time. 
An Indian textile company identified packing rejection of yarn cones as its major quality problem and decided to use six sigma methods to correct the problem (Arup Ranian Mukhopadhyay and Soumik Ray, 2006). The yarn cones were being rejected by customers due to unacceptable weight variation. The objective in the define phase is to reduce rejection of yarn cones through measuring the current performance level and initiating proper remedial action. Based on the data collected on packing rejection it was found that weight variation that is overweight and undenweight and those of sales turnover converged to the same vital few counts. In the measure phase data were collected from the final two steps of post spinning on count, empty cheese weight moisture content, length of yarn wound on to cone or cheese and the existing sigma level was calculated. Analysis was initiated to investigate the cause of weight variation on the basis of data collected. It was found that length of yarn wound onto cheese was the potential cause far variation in weight. In the second phase data were collected from assembly winding machines with $L M D$ attachments and no deficiency was found on repeatability. It was found that the inconsistent measurement of yarn length by LMDs attached with the assembly winding machines contributed towards the product variability. In the improve phase increasing the sensitivity of the performance of LMDs was achieved by implementing proper calibration procedures in some machines and by replacement of LMDs in other machines, which reduced cone weight variation substantially. In control phase to meet the farget value the length was set at some value using the regression analysis result.

Use of Principal Component Analysis: To demonstrate how statistical tools can evaluate the supplier's performance, the principal component analysis (PCA) is used (Fu-Kwun Wang et al., 2004). The PCA is a statistical method for multivariate data analysis that can be used to reduce the amount of data. It transforms original and related measurement variables into a set of orthogonal (uncorrelated) linear functions. Assume that $X$ is a $m \times n$ sample data matrix, where $m$ denotes the number of variables that are observed from a supplier and $n$ represents the number of suppliers being measured. $\overline{\mathrm{X}}$ is the sample mean of the observations, which is a $m$-vector value and $S$, a non singular $m \times m$ symmetric matrix, is the correlation matrix between observations. The spectral decomposition can be used to obtain, $\mathrm{D}=\mathrm{U}^{\mathrm{T}} \mathrm{SU}$ where $D$ is a diagonal matrix. The diagonal elements of $D$ are $\lambda_{1}, \lambda_{2}, \ldots, \lambda_{m}$, are the eigenvalues of $S$, and the columns of $U, u_{1}, u_{2}, \ldots, u_{m}$, are the eigenvectors of $S$. Consequently, the ith principal component $\left(\mathrm{PC}_{\mathrm{i}}\right)$ is

$$
\mathrm{PC}_{\mathrm{i}}=\mathrm{u}^{\mathrm{T}} \mathrm{x}, \quad \mathrm{i}=1,2, \ldots, \mathrm{m}
$$


where the $x^{\prime} s$ are $(m \times 1)$ vectors of the observations of the original variables. The proportion of variability associated with each principal component variable is,

$$
\mathrm{W}_{\mathrm{i}}=\frac{\lambda_{\mathrm{i}}}{\sum_{\mathrm{i}=1}^{\mathrm{m}} \lambda_{\mathrm{i}}}, \quad \forall \mathrm{i}=1,2, \ldots, \mathrm{m}
$$

However, only K principal components can contribute most of the system's variability. By using this subset, the multivariate quality characteristic problem can be reduced in dimension. Jackson (1980) proposes a test for identifying the significant components. It is,

$$
\chi^{2}=-(n-1) \sum_{j=k+1}^{m} \ln \lambda_{j}+(n-1)(m-k) \ln \frac{\sum_{j=k+1}^{m} \ln \lambda_{j}}{m-k}
$$

where $\chi^{2}$ has $r=\frac{1}{2}(m-k)(m-k+1)-1$ degrees of freedom.

The hypothesis that $\mathrm{H}_{0}: \lambda_{\mathrm{k}+1}=\lambda_{\mathrm{k}+2}=\ldots=\lambda_{\mathrm{m}}$ is applied against the alternatives when at least one has a different eigenvalue. Analysis of the loading and principal components requires that the principal components and their variables closely correspond to each other, that is, the angle between the vectors, represented as $R^{m}$, must be small. The correlation between the $i^{\text {th }}$ variable and the $i^{\text {th }}$ principal component is given by

$$
\rho_{\mathrm{ij}}=u_{\mathrm{ij}}\left[\sqrt{\frac{\lambda_{\mathrm{i}}}{S_{\mathrm{ii}}}}\right]
$$

where $\mathrm{u}_{\mathrm{ij}}$ denotes the loading for the ith observation in the ith principal component variable, $\lambda_{j}$ represents the eigenvalue associated with that principal component and $S_{\mathrm{ii}}$ is the variance of the ith variable. The single performance score by the first $k$ principal components is 


$$
\text { Performance Score }=\sum_{\mathrm{i}=1}^{\mathrm{k}} \mathrm{w}_{\mathrm{i}} \mathrm{PC}_{\mathrm{i}}
$$

The performance of a supplier can be measured by all the ratios of output and input measurement data relevant to the process improvement programs of a supplier. When multiple dimension are simultaneously considered in evaluating the overall competence of a supplier, the performance score of each supplier can be obtained by the PCA method. Suppliers with high performance scores are likely to sustain a high level of capabilities, and are better candidates for inclusion in an optimized supplier base. Thus, improvement in the quality of all supply chain processes reduces costs and improves the level of customer service.

Desirability function: Two steps were used in Harrington (1965) to calculate the desirability function. The first step concentrated on each criterion/response to assign an individual desirability. Criteria divided into two types - two sided criteria whose acceptable values were bounded by both an upper specification limit (USL) and a lower specification limit (LSL) and one sided criteria whose acceptable values were bounded by a single specification limit. For two sided criteria, the process of assigning desirability by calculating the scaled response value, $Y_{j}^{\prime}(x)$, was performed in Harrington using

$$
Y_{j}^{\prime}(x)=\frac{2 Y_{j}(x)-(U S L+L S L)}{(U S L-L S L)}
$$

Then, the user would need to input a single scaled criterion value for criterion $i$, $Y_{j, 0}^{\prime}$ and its assumed desirability $d_{0}$ (somehow independently of the values of other criteria). This pair of numbers $\left(Y_{j, 0}^{\prime}, d_{0}\right)$ was used to calculate the parameter $n$ using the formula.

$$
n=\frac{\ln \left[\ln \left(\frac{1}{d_{0}}\right)\right]}{\ln \left|Y_{j, 0}^{\prime}\right|}
$$

Further, the desirability for the two sided characteristic was obtained using 


$$
\mathrm{d}_{\mathrm{j}}\left(\mathrm{Y}_{\mathrm{j}}(\mathrm{x})\right)=\exp \left[\mathrm{Y}_{\mathrm{j}}^{\prime}(\mathrm{x})^{\mathrm{n}}\right]
$$

For single-sided criteria, the individual desirability measures or desirabilities were calculated as follows.

The engineer had to input two pairs $\left(Y_{i, 1}, d_{1}\right)$ and $\left(Y_{i, 2}, d_{2}\right)$ with the criteria values $Y_{i, 1}$ and $Y_{i, 2}$ given in a actual response units and it was assumed, without loss of generality, that $Y_{i, 1}>Y_{i, 2}$. Each of the response values was then scaled using the formula.

$$
Y_{j, 1}^{\prime}(x)=-\ln \left[-\ln \left(d_{1}\right)\right] \text { and } Y_{j, 2}^{\prime}(x)=-\ln \left[-\ln \left(d_{2}\right)\right]
$$

Then, the scaled criteria value, $Y_{j}^{\prime}(x)$, corresponding to the actual response $Y_{i}(x)$ was found through the following linear transformation.

$$
Y_{j}^{\prime}(x)=\left[\frac{\left(Y_{j}(x)-Y_{j, 2}\right)}{\left(Y_{j, 1}-Y_{j, 2}\right)}\right]\left[Y_{j, 1}^{\prime}(x)-Y_{j, 2}^{\prime}(x)+Y_{j, 2}^{\prime}(x)\right]
$$

Next, the desirability for the one sided characteristic was estimated using

$$
\mathrm{d}_{\mathrm{j}}\left(\mathrm{Y}_{\mathrm{j}}(\mathrm{x})\right)=\exp \left[-\exp \left(-\mathrm{Y}_{\mathrm{j}}(\mathrm{x})\right)\right]
$$

In the second stage of applying Harrington's method to estimate the system desirability, individual criteria desirabilities were combined using the following formula involving subjective weights of each of the criteria, $\mathrm{w}_{\mathrm{i}}$.

$$
D(x)=\left[d_{1}\left(Y_{1}(x)\right)^{w_{1}} \cdot d_{2}\left(Y_{2}(x)\right)^{w_{2}} \ldots . . d_{m}\left(Y_{m}(x)\right)^{w_{m}}\right]^{1 / s}
$$

where $s=\sum_{\mathrm{i}=1}^{\mathrm{m}} \mathrm{w}_{\mathrm{i}}$. Standard desirability functions result in the settings of process means some distance inside the specification limits. However, none of these functions has explicitly used the process standard deviation to determine the steepness of the functions and to regulate how far inside the limits the mean should be positioned. Each quality characteristic has both a mean, $\mu_{\mathrm{i}}$ and a standard deviation, $\sigma_{i}$, which may be regarded as properties or characteristics themselves associated with 
the engineering choices, $x_{1}, x_{2}, \ldots, x_{p}$. Another consideration is the concept of a shift of $\tau_{i} \sigma_{i}$ of the mean toward the relevant specification limit. In the six sigma literature, engineers are encouraged to consider the implications of a $1.5 \sigma_{i}$ shiff $\left(\tau_{\mathrm{i}}=1.5\right)$ in their design of products or processes.

For the case in which the individual characteristic, $i$, is bounded by upper and lower specification limits, USL and LSL respectively, and has associated mean, $\mu_{i}$, standard deviation, $\sigma_{i}$, and possible shift $\tau_{i}$, the proposed desirability function (Charles Ribardo and Theodore. T. Allen, 2003) is

$$
\mathrm{d}_{\mathrm{i}}\left(\mu_{\mathrm{i}}, \sigma_{\mathrm{i}}, \tau_{\mathrm{i}}\right)=\min \left[\text { Yield }_{\mathrm{i}}\left(\mu_{\mathrm{i}}, \sigma_{\mathrm{i}},+\tau_{\mathrm{i}}\right), \text { Yield }_{\mathrm{i}}\left(\mu_{\mathrm{i}}, \sigma_{\mathrm{i}},-\tau_{\mathrm{i}}\right)\right]
$$

where Yield ${ }_{i}\left(\mu_{i}, \sigma_{i}, s_{i}\right)=\phi\left[Z_{U S L, i}\left(\mu_{i}, \sigma_{i}, s_{i}\right)\right]-\phi\left[Z_{L S L, i}\left(\mu_{i}, \sigma_{i}, s_{i}\right)\right]_{\text {and }} \phi$ is the cumulative distribution function for unit normal random variables

where $=Z_{L S L, i}\left(\mu_{i}, \sigma_{i}, s_{i}\right)=\frac{1}{\sigma_{i}}\left[\operatorname{LSL}-\left(\mu_{i}+s_{i} \sigma_{i}\right)\right]$ and

$Z_{U S L, i}\left(\mu_{i}, \sigma_{i}, s_{i}\right)=\frac{1}{\sigma_{i}}\left[U S L-\left(\mu_{i}+s_{i} \sigma_{i}\right)\right]$

Defined in this way, the desirability $d_{i}\left(\mu_{i}, \sigma_{i}, \tau_{i}\right)$ corresponds to a conservative estimate of the long term fraction of conforming units under assumptions that are becoming increasingly standard. Yield $d_{i}\left(\mu_{i}, \sigma_{i},+\tau_{j}\right)$ corresponds to the yield under the assumption of a positive shift $\operatorname{Yield}_{\mathrm{i}}\left(\mu_{\mathrm{i}}, \sigma_{\mathrm{i}},+\tau_{\mathrm{i}}\right)$ and corresponds to the assumption of a negative shift. Taking the minimum of these two shifts may be regarded as conservative.

A new rating system is required in order to interpret the proposed desirability values. The decision maker must subjectively decide values of criteria are acceptable, good and target. Criteria $\mathrm{i}=1,2, \ldots, \mathrm{r}$ are associated with specification limits and yields, and the remaining $i=r+1, r+2, \ldots, m$ are characteristics for which values must be subjectively assigned. For combining the potentially several individual desirability measures into an overall desirability function, $\mathrm{D}(\mathrm{x})$, the proposed formula is, 
$\left[d_{1}\left(Y_{1}(x)\right) d_{2}\left(Y_{2}(x)\right) \ldots d_{r}\left(Y_{r}(x)\right)\right]^{w_{r}}\left[d_{r+1}\left(Y_{r+1}(x)\right)^{w_{r}+1} \cdot d_{r+2}\left(Y_{r+2}(x)\right)^{w_{r}+2} \ldots d_{m}\left(Y_{m}(x)\right)^{w_{m}}\right]^{/ s}$

where $s=\sum_{i=1}^{m} w_{i}$ and quantity in first bracket is an estimate of the overall process yield. The proposed desirability functions offer a concrete approach for extending the six sigma standard of quality to multi criterion design problems and conservatively estimates the effective yield under assumptions described in the $6 \sigma$ literature

\section{Discussion}

The 1.5 sigma shift resulting in a 3.4 DPMO level widely expounded in the $6 \sigma$ literature is a generally accepted assumption for manufacturing processes. This assumption has hardly been validated for services and transactional processes. Here, the applications considered are based on the above mentioned concept only. $6 \sigma$ methodology uses many existing statistical tools. So, there is no such analysis has been done with the alternative techniques. At the operational level, $6 \sigma$ projects would not create an impact if the requisite statistical concepts and tools are not effectively applied, therefore mastery of technical details is essential. To reach its goals, the implementation of a $6 \sigma$ program needs to change the way in which employees behave at work. $6 \sigma$ must alter how people actually behave at work and for this to happen, various behavioral and work processes are key to achieving these aspirations.

A greater attention by researchers to the following aspects in a $6 \sigma$ context would help management to develop a better understanding of performance and effectiveness.

a) Motivation, including leadership, learning and system constraints. b) Knowledge, skill and effort. As the scope of $6 \sigma$ application expands with time, more cross functional tools will be integrated with 60 to achieve even wider and deeper business performance improvement. There is an emerging trend of integrating artificial intelligence and information systems technologies, such as data mining, fuzzy logic and neural networks, into six sigma programs, in particular, design for six sigma (DFSS) for software. The 60 limits when applied to software development drives a software organization to achieve higher product quality. Combining the principles and tools of lean enterprise and six sigma provides an excellent way to improve the productivity and quality of providing financial services. Combining the advantages of speed and consistency, the service quality level could be substantially 
improved. These considerable benefits highlight the competences of the lean six sigma approach.

Six sigma can do more than just eliminate competitive disadvantages. If adds a whole range of new capabilities and skills to an organization. These skills could be described as scientific and disciplined problem solving and decision making behavior and effective distribution of information over the organization. These skills help tackling the general problem of economic planning under constraints to knowledge. 60 links customer requirements and process improvements with financial results while simultaneously providing the desired speed, accuracy and agility in today's e-age. 60 nowadays has become a quality philosophy and its knowledge has given lots of opportunities to statisticians working towards achieving the quality goals

\section{References}

1. Arup Ranjan Mukhopadhyay, Soumik Ray (2006), 'Reduction of Yarn packing defects using six sigma methods: A case study', Quality engineering No.18.

2. Charles Ribardo, Theodore. T. Allen (2003), An alsernative desirability function for achieving six sigma quality', Quality and Reliability Engineering International No.19.

3. Fu-Kwun Wang, Timon. C. Du, Eldon. Y. Li (2004), 'Applying six sigma to supplier development' Total Quality Management, Vol. 15 No. 9-10.

4. Gerald. J.Hahn, Necip Doganaksoy, Roger Hoerl (2000),'The evolution of six sigma' Quality Engineering No.12(3).

5. Harrington EC. (1965) 'The desirability function' Industrial Quality Control, 494 - 498.

6. Jackson. J.E. (980) 'Principal components and factor analysis: part 1 - principal components' Journal of Quality Technology, 12, pp. 201-213.

7. Ricardo Banuelas, Jiju Antony, Martin Brace (2005), 'An application of six sigma to reduce waste' Quality and Reliability Engineering International No 21.

8. Sunil Thawani (2004) 'Six sigma- Strategy for organizational excellence' Total Quality Management Vol.15 No.5.

9. Thong-Ngee Goh, Loon-Ching Tang, Shao-Wei Lam, Yin-Feng Gao(2006) 'Six sigma a SWOT analysis' Six Sigma and competitive advantage Vol. 2 No.3.

10. T.N. Goh, P.C. Low, K.L. Tsui, M. Xie (2003), 'Impact of six sigma implementation on stock price performance' TQM and Business excellence Vol. 14 No.7. 